\title{
Environment and unconditioned motor behavior: Influences of drugs and environmental geometry on behavioral organization in rats
}

\author{
MARTIN P. PAULUS and MARK A. GEYER \\ University of California, San Diego, La Jolla, California
}

\begin{abstract}
Unconditioned motor behavior is widely used as a behavioral assay for a variety of experimental manipulations. This investigation examined the relationships in rats between effects of drugs $(5.0 \mathrm{mg} / \mathrm{kg}$ of nomifensine, $5.0 \mathrm{mg} / \mathrm{kg}$ of MBDB, and $0.40 \mathrm{mg} / \mathrm{kg}$ of heroin) and the geometric properties of the testing environment (a $30.5 \times 60.5 \mathrm{~cm}$ rectangular chamber, a 40-cm-diam circular chamber, and a $60.5 \times$ $60.5 \mathrm{~cm}$ square chamber) using a video-camera-based observation system on measures characterizing the geometrical characteristics of movements. The results show that spatial patterns of movements differ significantly across environments having different geometric properties. The magnitude of drug-induced changes of these patterns can be proportional or inversely proportional to the baseline rate observed in saline animals. These results suggest that, relative to the performance of saline controls, the effect of a drug can be characterized as environmentally dependent, environmentally independent, or environmentally idiosyncratic on the basis of its effect in different environments.
\end{abstract}

\begin{abstract}
Assessment of rodent locomotor activity has become one of the most widely used assays to determine the behavioral effects of various experimental manipulations ranging from genetic changes, for example knock out mice, to pharmacological challenges, for example amphetamineinduced locomotor activity (Creese \& Iversen, 1974; Eilam \& Golani, 1990; Geyer, 1982; Lat, 1965; Paulus \& Geyer, 1991). This wide range of applications is due largely to the fact that experimental paradigms based on unconditioned motor activity probe a variety of behaviors, can be recorded automatically, and can quickly generate an effect profile (Denenberg, 1969; McReynolds, 1962; Robbins, 1977). For example, locomotor activity has been used to discriminate drug effects (Geyer, Russo, \& Masten, 1986; Ljungberg \& Enquist, 1987; Szechtman, Ornstein, Teitelbaum, \& Golani, 1985), to illuminate the functional significance of specific neuroanatomical sites (Creese \& Iversen, 1974), or to screen drugs for potential psychoactivity (Paulus \& Geyer, 1992). Nevertheless, unconditioned motor behavior comprises a wide variety of different behaviors in rodents, ranging from exploratory elements to stereotypic or perseverative behaviors (Donat, 1991; Montgomery, 1955; Schiorring, 1971; Teitelbaum, Szechtman, Sirkin, \& Golani, 1982). Thus, the observed behavior results from a combination of different individual behavioral elements.
\end{abstract}

This work was supported by an NIDA grant (DA02925) to M.A.G. and an NIDA grant (DA11277) to M.P.P. The invaluable help of Virginia Masten and Elizabeth Lutz in this undertaking is gratefully acknowledged. Correspondence should be addressed to M. P. Paulus, Department of Psychiatry, School of Medicine, University of California, San Diego, La Jolla, CA 92093-0804 (e-mail: martin@rat.ucsd.edu).
Numerous investigators have recognized the necessity for a pattern analysis and proposed different approaches to quantify the various components of locomotor behavior (Eilam \& Golani, 1990; Geyer et al., 1986; Ljungberg \& Enquist, 1987; Nickolson, 1981; Sanberg et al., 1987). For example, some investigators have rated videotaped movement sequences; others have defined locomotor "trips" and applied different statistics to quantify these components (Mueller, Hollingsworth, \& Cross, 1989; Teitelbaum et al., 1982). Several groups have used a large number of measures to comprehensively describe locomotor activity (Geyer et al., 1986; Sanberg et al., 1987). Alternatively, we have developed a technique to quantify sequences of movements in order to assess patterns of locomotor activity (Paulus, Callaway, \& Geyer, 1993; Paulus \& Geyer, 1991, 1993a). This approach was based on the assumption that behavior consists of purposefully organized microevents resulting in distinct patterns of activity. For example, exploratory activity consists of consecutive straight movements to reach a location in the environment combined with circumscribed movements to further process localized sensory stimuli. This assumption parallels the descriptive approaches to locomotor behavior that have been proposed by various investigators (Eilam \& Golani, 1990; Geyer, 1982; Teitelbaum et al., 1982). However, our approach is not based on the assumption of distinct behavioral categories implicit in the use of experimenter-defined responses. Instead, patterns are thought to emerge from the geometrical characteristics of the sequences of movements.

This approach was derived from the work of a large number of mathematicians and theoretical physicists in the fields of fractal geometry (Higushi, 1988; Mandelbrot, 1977), ergodic theory (Ruelle, 1984), nonlinear dynamics 
(Grassberger, Badii, \& Politi, 1988), and complex physical systems (Stanley, 1987). Fundamental to these approaches is the notion that the dynamical and geometrical characteristics of a system can be quantified by measuring the system's behavior on different spatiotemporal scales. Specifically, the distance between consecutive movements of an animal serves as a simple measure to quantify the amount of activity. Such a measure inherently depends on the spatial or temporal scale chosen to measure the distance. For example, an animal that explores one area of the enclosure exhibits a large amount of activity on a small scale, that is, the local area of the enclosure, but not on a large scale, the entire enclosure. Recently, it has been suggested that, for biological systems, there may be an invariant relationship between the dynamical characteristics, in this case the predictability of a movement pattern, and the geometrical characteristics, here the geometry of the movement patterns (Mandell \& Selz, 1997). Thus, this approach may yield important insights into the organization of biological systems in general and of rat motor behavior in particular.

This approach allowed for a comprehensive assessment of sequences of behavior and significantly extended previous analyses using transition matrices or information statistics (Geyer, 1982; Norton, 1977). The utility of this approach has been confirmed by several findings. First, geometrical patterns of movements are linearly independent of the amount of activity exhibited by rats in a rectangular enclosure (Paulus \& Geyer, 1993b). Second, drugs that similarly affect the amount of activity differentially affect the geometrical patterns of movements (Geyer \& Callaway, 1994; Geyer et al., 1986; Paulus \& Geyer, 1992). Third, drugs with similar mechanisms of actions generate similar geometric patterns (Paulus et al., 1993). Fourth, subtle differences of drug effects in humans can be related to subtle differences in changes of geometrical patterns induced by these drugs (Paulus et al., 1993; Paulus \& Geyer, 1992). Fifth, circumscribed lesions of dopaminergic systems involved in generating varied locomotion versus stereotypy result in corresponding geometrical pattern changes. These results suggest that measures of geometrical patterns reflecting behavioral organization of individual movements to form coherent behavioral acts provide sensitive and independent information about unconditioned motor behavior.

The above results were obtained using a rectangular enclosure and a photobeam-based system, the so-called behavioral pattern monitor (BPM) (Geyer et al., 1986). Locomotor activity has been assessed in different types of enclosures, most frequently using rectangular, circular, and square geometries. Implicit or explicit in comparing the results from studies of locomotor behavior is the assumption that the behavioral effects are relatively invariant with respect to the geometry of the enclosure. More appropriately, however, unconditioned motor activity can be conceptualized as an emerging behavior resulting from a variety of significant influencing factors, including the intrinsic behavioral repertoire, the experimental manipulation, and the characteristics of the environment. Some investigators have established that rats organize the exploration of their environments in relationship to a home area that is typically located close to a corner of the enclosure (Adams \& Geyer, 1982; Eilam \& Golani, 1990). These observations suggest that the geometry of the enclosure influences the geometrical organization of movements. Moreover, previous investigations have demonstrated clearly that the characteristics of experimental manipulations affecting behavior depend on the baseline frequency of distinct behavioral categories (Robbins, 1981; Schiorring, 1971). This finding has been called "rate dependency" or "probability dependency" (Robbins, 1981) and has also been extended neurobiologically (Weiss, Paulus, Lorang, \& Koob, 1992). Therefore, it is reasonable to assume that patterns of activity are affected not only by the drug's particular mechanism of action, but also by the geometry of the enclosure, the baseline activity, and the baseline patterns of activity.

The effect of experimental manipulation on changes in behavior is a function of the baseline probability or frequency (rate) of the behavior. Moreover, intrinsic behavioral organization and external environmental influences determine this baseline rate. Thus, the following hypotheses were proposed. First, the organization of unconditioned locomotor behavior depends on the environmental characteristics. Second, the organization of unconditioned locomotor behavior is affected differentially by drugs, depending on the environmental characteristics. Third, the overall characteristic of the drug effect is similar in geometrically distinct environments. To test these hypotheses, a video-camera system was used to obtain detailed recordings of the movement sequences of rats in three geometrically different environments. The organization of unconditioned locomotor behavior was assessed using the fluctuation spectrum of local spatial scaling exponents, as detailed elsewhere (Paulus et al., 1993; Paulus \& Geyer, 1993a). Three pharmacological probes known to generate distinct behavioral organizations in a photocell system were used to manipulate the movement patterns. First, nomifensine, an indirect dopaminergic agonist, induces a significant increase in the amount of motor activity and a more uniform geometrical pattern consisting of predominantly meandering movement sequences (Paulus et al., 1993). Second, $\mathrm{MBDB}$, an indirect serotonergic agonist, results in a significant shift of the geometrical pattern toward straight or directed movements associated with an increase in the amount of motor activity (Callaway, Johnson, Gold, Nichols, \& Geyer, 1991; Paulus \& Geyer, 1992). Third, heroin, an opioid agonist, induces a locomotor pattern similar to that induced by MBDB but associated with a reduction in the amount of activity (unpublished observations). The extent of local pattern differences was used as the primary dependent variable to determine the effect of environment and drugs. 


\section{METHOD}

Three separate experiments were conducted. For each experiment, rats were exposed only once to the apparatus, and the testing was performed during the animals' dark-cycle or high-activity period.

\section{Animals and Apparatus}

For each experiment, 32 (circular and square enclosures) or 36 (rectangular enclosure) male Sprague-Dawley rats, weighing 250-300 g (Simonsen Laboratories, Gilroy, CA, or Harlan, Indianapolis), were kept on a reverse 12:12-h light:dark cycle and allowed to acclimate to the animal facilities for at least 7 days before behavioral testing. The animals were brought in groups of 3 to the laboratory $1 \mathrm{~h}$ prior to testing. Each animal was tested only once in the Video Tracker. Once brought to the laboratory, the animals were kept in a dark room under a black cloth until testing. The rats received injections s.c. $10 \mathrm{~min}$ prior to exposure to the enclosure. At that time, the rats were marked with a small, fluorescent, water-based paint spot between the scapulae to improve tracking of the animals with the video camera.

The following drugs were tested: nomifensine (RBI, Natick, MA), $5.0 \mathrm{mg} / \mathrm{kg}$; MBDB (D. Nichols, Purdue University), $5.0 \mathrm{mg} / \mathrm{kg}$; and heroin (NIDA), $0.40 \mathrm{mg} / \mathrm{kg}$. The drugs were prepared immediately before each experiment, dissolved in saline, and injected s.c. $10 \mathrm{~min}$ prior to exposure in the Video Tracker. The doses of the drugs had been selected on the basis of prior test results obtained in the BPM (Callaway et al., 1991; Paulus et al., 1993; Paulus \& Geyer, 1992). After each testing session, which lasted $30 \mathrm{~min}$, the enclosure was cleaned thoroughly. The duration of testing had been determined from prior testing of these compounds in the BPM, which had resulted in characteristic locomotor patterns and/or significant changes in the amount of activity.

The data-acquisition system consists of three components. (1) The Video Tracker enclosure, a $60.5 \times 60.5 \times 50 \mathrm{~cm}$ plastic box that is surrounded by a larger wooden box equipped with a fan generating white noise to mask outside sounds. The animal movements are recorded by a video camera mounted in the ceiling of the enclosure. Three 20-cm-long black lights are mounted in the front middle and back of the ceiling to ensure equal but low visible lighting throughout the chamber. Two small vents are located in the middle left and right side of the ceiling to facilitate a constant air flow. (2) The Chromotrack (San Diego Instruments, San Diego) hardware is used to digitally process the signal coming from the video camera. This processor provides the $x, y$ coordinates of the left and uppermost spot exceeding a manually adjustable signal threshold. For optimal tracking, this threshold was adjusted manually before each experiment by minimizing the area on the back of the rat that was tracked by the camera and eliminating the loss of tracking due to belowthreshold camera signals. An error count of lost signals during testing confirmed that tracking was lost in less than $5 \%$ of the time. (3) A PC-compatible computer continuously monitored the Chromotrack hardware and stored the $(x, y)$ positions with a spatial resolution of $0.3 \mathrm{~cm}$ and a temporal resolution of $18.18 \mathrm{msec}(55 \mathrm{~Hz})$. The raw data were stored as binary files on the computer. Subsequently, these raw-data files were translated into ASCII files at the end of each experiment.

Sequences of microevents defined by $(x, y, t)$ coordinates, that is, the $x$ and $y$ location of the animal as well as the time spent at that location, were extracted from the raw data. A distinct microevent consisted of an $(x, y)$ position separated by at least $1.5 \mathrm{~cm}$ from the previous microevent and an event duration of at least $36.36 \mathrm{msec}$. These values were selected to compare microevent sequences obtained from the Video Tracker with those obtained from the BPM. The sequences of microevents for the first $30 \mathrm{~min}$ in the enclosure were used to quantify the amount of activity and the geometry of movements.

\section{Video Tracker Environments}

Three different environments were selected for testing on the basis of size (small, medium, large), symmetry (low, medium, high), and boundary characteristics (variable, uniform, constant). (1) A $30.5 \times 60.5 \mathrm{~cm}$ rectangular environment (area $1,845 \mathrm{~cm}^{2}$ ) was used that was equal in size to the enclosure of the BPM characterized by a medium-sized, low-symmetry, and variable boundaries. (2) A circular environment with $40-\mathrm{cm}$ diameter (area $1,256 \mathrm{~cm}^{2}$ ) characterized by a small-sized, high-symmetry, and constant boundary was constructed from a black, plastic can, which was inserted and affixed firmly in the Video Tracker box. (3) The entire inner enclosure of the Video Tracker was used as a square environment with a side length of $60.5 \mathrm{~cm}\left(3,660 \mathrm{~cm}^{2}\right)$ characterized by large-sized, medium-symmetry, and uniform boundaries.

\section{Behavioral Assessment of Motor Activity \\ and Geometrical Patterns of Movements}

The behavioral assessment of the animals is based on ensembles of microevents $(x, y, t)$ in the different environments. For the assessment of gross motor activity, a microevent was defined as a movement exceeding 5 pixel units in either $x$ or $y$ direction. The spatial resolution of the Video Tracker was decreased to eliminate the contribution of very fine motor movements when quantifying gross motor activity. The amount of motor activity was measured by the number of microevents, counts, during the $30-\mathrm{min}$ exposure to the enclosure and evaluated in 5-min bins.

We have previously proposed a scaling hypothesis (Paulus \& Geyer, 1991) for the quantitative assessment of spatial and temporal patterns of movement sequences. This hypothesis is based on the notion that behavioral microevents lack a characteristic spatial or temporal scale. Therefore, patterns of behavior are best described by measures that capture the behavior across a range of spatial and temporal scales. In particular, the distance traveled between $k$ microevents is related to the number of distinct microevents, $k$. Here, $k$ corresponds to the resolution with which the behavior is observed. Using this hypothesis, straight, meandering, or circumscribed movements can be distinguished readily and quantitatively on the basis of their scaling characteristics.

The particular functional form relating the distance traveled to the number of microevents is derived from scaling approaches in physical systems. The applicability and utility of these approaches have been demonstrated in a large number of complex physical and biological systems (Feder, 1988; Stanley, 1987). Geometrically, scaling approaches are closely related to the quantification of a geometrical object by its fractal dimension (Mandelbrot, 1977). This functional form, as applied to the pattern analysis of movements, reads as follows:

\section{[Distance traveled] ([between $k$ microevents]) $\propto m^{\text {scaling exponent }}$}

[Distance traveled] ([between $m \times k$ microevents]).

Thus, the scaling relationship is described quantitatively by the spatial scaling exponent, $d$.

The spatial scaling exponent quantifies the overall geometry of movements. However, patterns of rat movements comprise a highly organized assembly of straight-directed, meandering, and circumscribed movements. This assembly cannot always be quantified sufficiently by the average scaling relationship. Stated simply, an average number calculated from organized sequences of straight, meandering, or circumscribed movements does not adequately describe the complexity of the behavior. Therefore, the notion of an extended scaling hypothesis was introduced to complement the characterization of the overall geometry of the movements. The extended scaling hypothesis states that organized sequences of straight, meandering, or circumscribed movements are more adequately quantified by a range of local scaling exponents, $d_{i}$. The frequency of these local scaling exponents is quantified by a scale-independent density 
function which has been called the fluctuation spectrum of local spatial scaling exponents:

and is defined by

[Number of] ([local scaling exponents characterizing distances between $k$ microevents $]) \propto k^{f(\text { local scaling exponent }])}$.

For the analysis of rat movements, the Euclidean distance, $\Delta$, between two microevents is calculated for different segments, $i$, at different levels of resolution, $k$. In addition, the distances for different $k$ are averaged within each segment, $i$, to improve the estimation of $d_{i}$. The number of microevents comprising a segment was optimized between the following competing tendencies. While segments consisting of few microevents can be used to differentiate even transient movement patterns, segments consisting of a large number of microevents yield more accurate least squares fits for the different $d_{i}$. Extensive variations of these parameters followed by numerical analysis resulted in the selection of a segment size of 16 microevents and the resolution range $k=1$ to 12 .

For practical calculations, several groups have advocated the use of a partition function (Halsey, Jensen, Kadanoff, Procaccia, \& Shraiman, 1986), which corresponds to a "filtered summation" of the distance traveled for the microevent segments. A simple transformation of this partition function for different values of the filter parameter was used to calculate both the local spatial scaling exponents and the fluctuation spectrum of local spatial scaling exponents (for details, see Paulus \& Geyer, 1993a).

\section{Statistical Comparison}

Differences in movement patterns between the treatment groups and saline animals or between treatment groups in different environments were based on group-average $d$ and the $f(d)$ functions. The significances of these differences were quantified by a modified $z$-transformed normal score. The significance of local pattern differences was determined by $Z$-scores of $\left|f_{z, \lambda}(d)\right|>2.0$.

Within a given environment, the effect of drugs on amount of activity or the average $d$ measure was evaluated statistically using a repeated analysis of variance (ANOVA) model (drug group $\times$ time). All $F$ ratios and their associated probabilities are reported.

\section{RESULTS}

\section{Experiment 1: Thirty-Minute Exposure to the Rectangular Chamber}

Activity. First, the level of motor activity significantly attenuated during a $30-\mathrm{min}$ exposure to the rectangular chamber $[F(5,150)=52.99, p<.00001]$. Second, animals treated with saline, nomifensine $(5.0 \mathrm{mg} / \mathrm{kg}), \mathrm{MBDB}$ $(5.0 \mathrm{mg} / \mathrm{kg})$, or heroin $(0.4 \mathrm{mg} / \mathrm{kg})$ differed significantly in the amount of motor activity exhibited during the 30 min interval in the rectangular chamber $[F(1,30)=$ $26.48, p<.00001]$. Third, the ANOVA revealed a significant treatment $\times$ time interaction $[F(15,150)=4.19$, $p<.0001$ ] , suggesting that the different treatments resulted in distinct habituation curves of motor activity. For example, relative to saline controls, $\mathrm{MBDB}$ and heroin reduced the amount of activity during the first 5-10 $\mathrm{min}$ and did not change or increase levels of activity in subsequent time blocks. In contrast, nomifensine increased the amount of motor activity across all time points and resulted in a habituation curve similar to that observed with saline-control animals (Figure 1a).

Geometrical patterns of activity, $\boldsymbol{f}(\boldsymbol{d})$. Movement patterns of rats treated with $5.0 \mathrm{mg} / \mathrm{kg}$ of nomifensine were characterized by a significantly reduced contribution $\left[f_{z}(d) \approx-8.0\right]$ of straight movements $\left(1.0 \leq d_{i} \leq 1.25\right)$ when compared with saline controls. In contrast, the contribution of highly circumscribed movements ( $1.5 \leq$ $d_{i} \leq 2.0$ ) was elevated significantly in nomifensine animals $\left[f_{z}(d) \approx 4.0\right]$ compared with controls. Thus, rats treated with nomifensine displayed an overall shift toward local movements. The nomifensine-induced change in individual movement patterns resulted in an overall shift toward circumscribed movements, as indicated by a significantly increased average $d=1.346 \pm 0.03$ compared with control animals $(d=1.240 \pm 0.04)$ (Figures $\mathrm{lb}$ and $\mathrm{lc}$ ).

Movement patterns of rats treated with $5.0 \mathrm{mg} / \mathrm{kg}$ of MBDB were characterized by an increased contribution $\left[f_{z}(d) \approx 2.0\right]$ of straight $\left(1.0 \leq d_{i} \leq 1.25\right)$ without significant changes in local movements relative to saline controls. However, the effect of MBDB on straight movements did not result in an overall change of movement patterns, as indicated by an average $d=1.231 \pm 0.01$, which did not differ significantly from that of saline controls.

Movement patterns of rats treated with $0.4 \mathrm{mg} / \mathrm{kg}$ of heroin were characterized by a slight $\left[f_{z}(d) \approx-1.0\right]$, but not significantly reduced, contribution of straight movements $\left(1.0 \leq d_{i} \leq 1.25\right)$ compared with control animals. In contrast, local movements $\left(1.5 \leq d_{i} \leq 2.0\right)$ were elevated $\left[f_{z}(d) \approx 6.0\right]$ relative to those of controls. Nevertheless, the average spatial scaling exponent, $d=1.325 \pm$ 0.018 , was not affected significantly by heroin.

\section{Experiment 2: Thirty-Minute Exposure to the Circular Chamber}

Activity. The amount of activity exhibited by animals treated with $5.0 \mathrm{mg} / \mathrm{kg}$ of nomifensine, $5.0 \mathrm{mg} / \mathrm{kg}$ of MBDB, or $0.4 \mathrm{mg} / \mathrm{kg}$ of heroin changed significantly over time $[F(5,150)=29.13, p<.0004]$. The amount of activity was affected differentially by the treatments $[F(3,28)=8.23, p<.0004]$. Moreover, a significant treatment $\times$ time interaction $[F(5,140)=5.39, p<.00001]$ indicated that the drugs differentially affected the habituation of activity. Specifically, while nomifensine-treated animals displayed elevated activity levels throughout the 30-min interval, heroin reduced the amount of activity during the first $10 \mathrm{~min}$ in the circular chamber and subsequently increased slightly the amount of activity relative to that of saline controls. MBDB-treated rats exhibited activity levels comparable to those of saline controls throughout the entire interval (Figure 1d).

Geometrical patterns of activity, $f(d)$. Nomifensine treatment resulted in a strongly reduced contribution $\left[f_{z}(d) \approx-6.0\right]$ of highly directed movement sequences $\left(1.0 \leq d_{i} \leq 1.25\right)$. This reduction was accompanied by a reduced contribution of highly local movements $(1.5 \leq$ $d_{i} \leq 2.0$ ). Thus, these animals engaged predominantly in meandering movement patterns in the circular chamber with local scaling exponents in the midrange of the $f(d)$ function. The meandering path pattern was associated with an average spatial scaling exponent $(d=1.321 \pm$ 0.02 ), which was not significantly higher than the spatial 

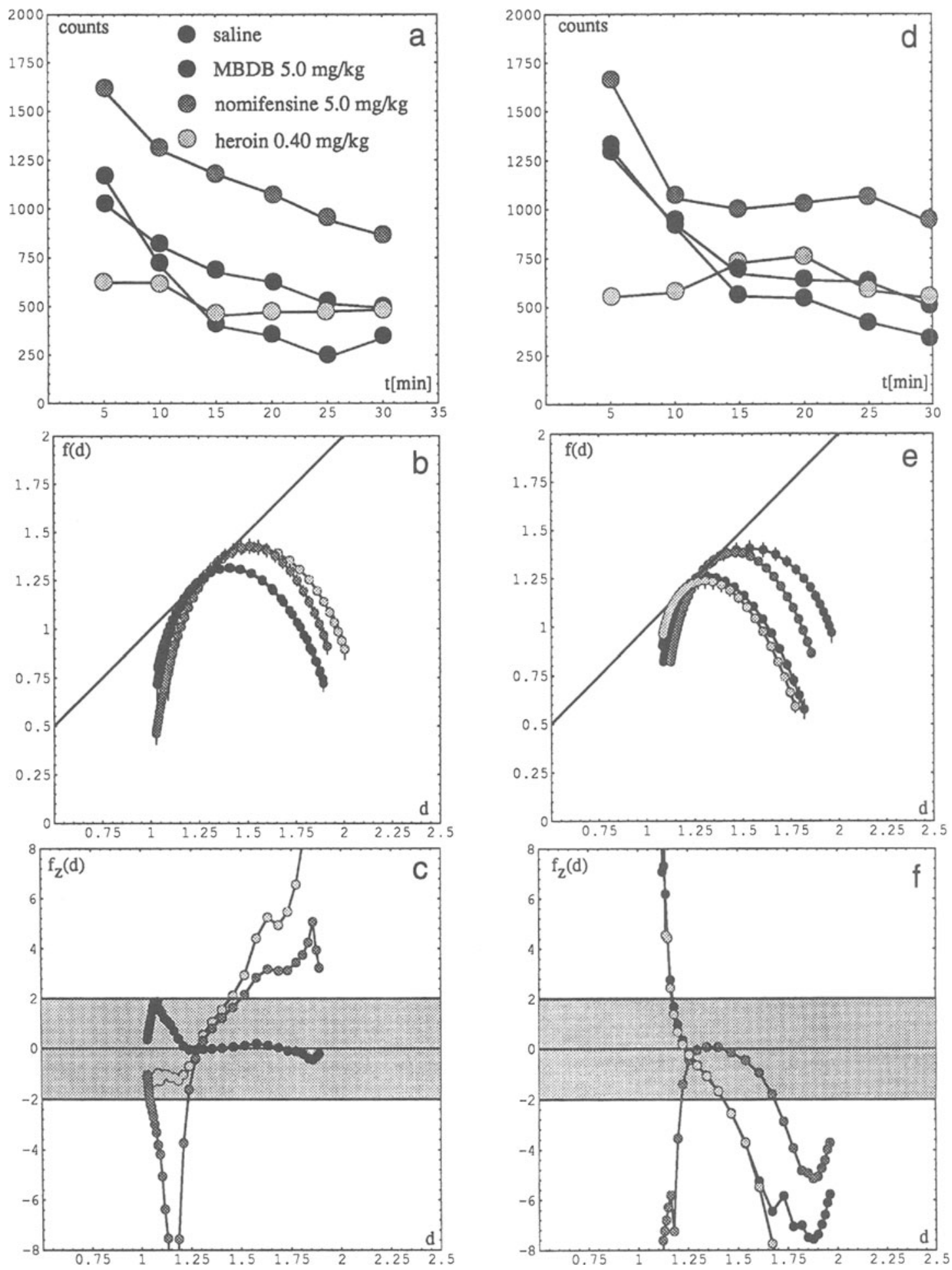

Figure 1. This figure shows the effect of saline, $5.0 \mathrm{mg} / \mathrm{kg}$ of $\mathrm{MBDB}, 5.0 \mathrm{mg} / \mathrm{kg}$ of nomifensine, and $0.4 \mathrm{mg} / \mathrm{kg} \mathrm{of} \mathrm{heroin}$ on counts $(\mathrm{a}, \mathrm{d})$, the fluctuation spectrum of local spatial scaling exponents $(\mathrm{b}, \mathrm{e}), f(d)$, and the local pattern differences $f_{z}(d)(c, f)$ in the rectangular chamber $(a, b, c)$ and circular chamber $(d, e, f)$. 
scaling exponent for saline animals $(d=1.295 \pm 0.02)$ (Figures 1e and 1f).

Both straight-directed movement sequences $(1.0 \leq$ $\left.d_{i} \leq 1.25\right)$ and local movements $\left(1.5 \leq d_{i} \leq 2.0\right)$ were strongly affected by $5.0 \mathrm{mg} / \mathrm{kg}$ of MBDB. While straightmovement sequences contributed significantly more $\left[f_{z}(d)>8.0\right]$ to the overall path pattern, highly local movements were found to contribute significantly less to the movement patterns when compared $\left[f_{z}(d) \approx-6.0\right]$ with saline controls. This shift in both subsets of the geometrical movement patterns led to an overall change of the geometrical movement characteristics toward straightmovement sequences characterized by an average spatial scaling exponent $(d=1.205 \pm 0.02)$ that differed significantly from that of saline controls.

In the circular chamber, $0.4 \mathrm{mg} / \mathrm{kg}$ of heroin resulted in changes of geometrical movement patterns similar to those observed after MBDB treatment. The contribution of both straight sequences $\left(1.0 \leq d_{i} \leq 1.25\right)$ and local movements $\left(1.5 \leq d_{i} \leq 2.0\right)$ differed significantly from the contributions of saline controls. Heroin-treated rats exhibited significantly more straight movements $\left[f_{z}(d)>\right.$ $8.0]$ and significantly fewer circumscribed movements $\left[f_{z}(d) \approx 8.0\right]$ than the control animals. This dramatic change in movement patterns resulted in an overall shift of the geometrical characteristics toward straight movements, as indicated by a significantly reduced average spatial scaling exponent $(d=1.186 \pm 0.02)$.

\section{Experiment 3: Thirty-Minute Exposure to the Square Chamber}

Activity. Levels of motor activity in the square chamber exhibited by rats treated with $5.0 \mathrm{of} \mathrm{mg} / \mathrm{kg}$ of nomifensine, $5.0 \mathrm{mg} / \mathrm{kg}$ of MBDB, or $0.4 \mathrm{mg} / \mathrm{kg}$ of heroin changed significantly over time $[F(5,140)=65.26, p<$ $.00001]$. These treatments differed significantly from each other $[F(3,28)=5.81, p<.0032]$. Moreover, the significant effect of a treatment $\times$ time interaction $[F(5,140)=$ $4.29, p<.00001]$ pointed toward a differential change of the amount of activity over time by these drugs. Specifically, nomifensine significantly elevated motor activity levels throughout the 30-min test. Both MBDB and heroin initially reduced the amount of activity but elevated the activity above saline control levels during the last $15 \mathrm{~min}$ in the square chamber (Figure 2a).

Geometrical patterns of activity, $\boldsymbol{f}(\boldsymbol{d})$. Nomifensine treatment changed the contributions of straight and local movements to the overall geometrical pattern of activity. Specifically, straight movements $\left(d_{i} \approx 1.1\right)$ were reduced significantly $\left[f_{z}(d) \approx-6.0\right]$ relative to those of saline controls. Simultaneously, local movements $\left(1.0 \leq d_{i} \leq\right.$ 1.5) were increased significantly above those of saline levels $\left[f_{z}(d) \approx 7.0\right]$. However, these local changes did not significantly affect the overall geometrical characteristics of the behavior indicated by the average spatial scaling exponent $(d=1.226 \pm 0.03$ ) (Figures $2 \mathrm{~b}$ and $2 \mathrm{c}$ ).

MBDB-treated animals displayed only a slight and nonsignificant change in straight movements relative to those of saline control animals. However, local-movement
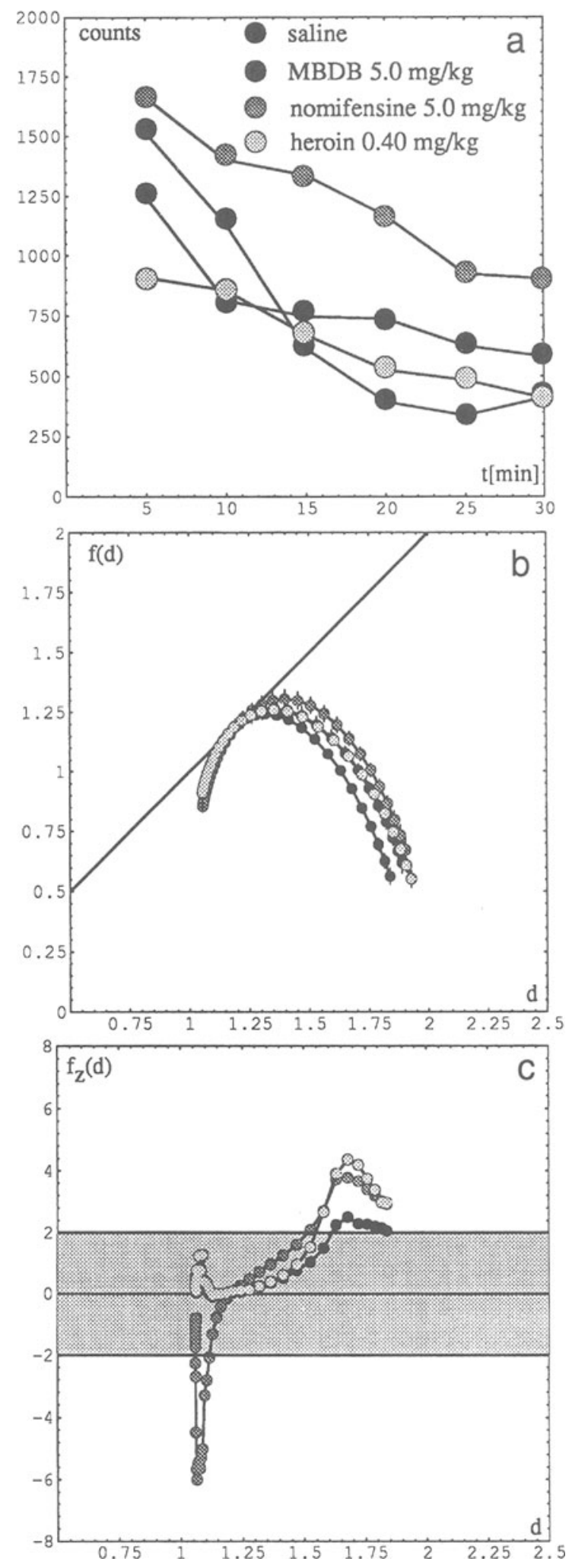

Figure 2. This figure shows the effect of saline, $5.0 \mathrm{mg} / \mathrm{kg}$ of MBDB, $5.0 \mathrm{mg} / \mathrm{kg}$ of nomifensine, or $0.4 \mathrm{mg} / \mathrm{kg}$ of heroin on counts (a), the fluctuation spectrum of local spatial scaling exponents (b), $f(d)$, and the local pattern differences $f_{z}(d)$ (c) in the square chamber. 
sequences $\left(1.5 \leq d_{i} \leq 2.0\right)$ were elevated significantly $\left[f_{z}(d) \approx 7.0\right]$ relative to those of controls. Thus, the geometrical structure of local movement sequences was shifted toward an increase in local movements. Nevertheless, these effects were not sufficient to significantly affect the average spatial scaling exponent $(d=1.191 \pm$ .03 ) relative to that of controls.

The effect of heroin treatment on the geometrical path structure of the animals was similar to the effects of nomifensine and MBDB. Specifically, a slight increase in the contribution of straight-movement sequences was accompanied by a significant elevation $\left[f_{z}(d) \approx 4.0\right]$ of local movements $\left(1.5 \leq d_{i} \leq 2.0\right)$.

\section{Comparisons Between Chambers}

Tables 1 and 2 summarize the results of the geometrical pattern differences, that is, the drug-induced changes in patterns of movements in one environment relative to another environment and the differences between control groups in the three environments. In particular, Table 2 shows that the contributions of straight, meandering, or circumscribed movements are affected differentially by each treatment in the square, rectangular, or circular environments, suggesting that an environment-specific behavioral profile contributes importantly to the geometrical path structure. Moreover, Figure 3 shows exemplary animal-movement patterns after saline or nomifensine treatment in the rectangular, circular, or square chambers.

Comparing saline controls across different chambers revealed that contributions of straight movements (low $d$ values) increased and that of meandering (mid $d$ ) and circumscribed $(\mathrm{mid} d)$ movements decreased in the square and rectangular chambers relative to the circular chamber. While saline animals did not differ in the contribution of straight movements between the square and

Table 1

z-Score Differences: Drug Versus Control for Straight, Meandering, and Local Movements

\begin{tabular}{lccr}
\hline Movements & Nomifensine & MBDB & Heroin \\
\hline \multicolumn{4}{c}{ Square Chamber } \\
Low $d$ & -1.50 & 0.10 & 0.22 \\
Mid $d$ & 2.09 & 1.15 & 1.88 \\
High $d$ & 3.16 & 2.19 & 3.31 \\
& \multicolumn{4}{c}{ Rectangular Chamber } \\
Low $d$ & -5.37 & 0.85 & -1.10 \\
Mid $d$ & 1.85 & 0.04 & 2.91 \\
High d & 3.92 & -0.26 & 9.43 \\
& \multicolumn{4}{c}{ Circular Chamber } \\
Low $d$ & -8.05 & 4.83 & \\
Mid $d$ & -0.74 & -3.34 & -3.79 \\
High $d$ & -4.54 & -7.02 & -10.66 \\
\hline
\end{tabular}

Note-This table summarizes the differences between nomifensine, MBDB, or heroin and saline control animals for the contribution of low $d(d<1.25)$, midrange $d(1.25 \leq d<1.75)$, and high $d(d>1.75)$ describing straight, meandering, and highly circumscribed movements, respectively. $z$ scores are obtained by calculating the differences between the $f(d)$ value for the drug and control divided by the averaged standard deviation.
Table 2

z-Score Differences for Drugs in Different Environments

\begin{tabular}{lccrr}
\hline Movements & Saline & Nomifensine & MBDB & Heroin \\
\hline & Square Versus & Rectangular & Chamber \\
Low $d$ & 1.61 & 5.62 & 0.95 & \\
Mid $d$ & -7.73 & -1.31 & -0.99 & -3.19 \\
High $d$ & -5.80 & -2.66 & -1.51 & -8.03 \\
\multicolumn{5}{c}{} \\
Low $d$ & Square Versus Circular Chamber \\
Mid $d$ & 5.14 & 3.74 & 2.29 & 0.96 \\
High $d$ & -4.50 & -1.03 & 0.90 & 2.16 \\
& -8.31 & -1.25 & 2.15 & 5.51 \\
Low $d$ & Rectangular Versus Circular Chamber & \\
Mid $d$ & 4.49 & 3.89 & 1.78 & -1.05 \\
High $d$ & -2.00 & 0.65 & 2.78 & 4.92 \\
\hline
\end{tabular}

Note-This table shows the comparative differences in contributions of straight, meandering, and circumscribed movements for a drug in the square, rectangular, or circular chambers, respectively. $z$ score calculations are analogous to those described in Table 1.

the rectangular chambers, these animals exhibited significantly fewer meandering and circumscribed movements in the square than in the rectangular chamber. Comparing nomifensine treatment across different chambers showed that straight movements were more significantly affected in the square and rectangular chamber than in the circular chamber. In contrast, circumscribed movements were more significantly affected in the square and circular chamber than in the rectangular chamber. Comparing MBDB treatment across different chambers showed that straight movements were more significantly affected in the square chamber than in the circular chamber. MBDB's effect on circumscribed movements was more significant in the square and rectangular chambers than in the circular chamber. Finally, heroin treatment resulted in a graded effect on circumscribed movements across different chambers. First, there was a significantly increased contribution of circumscribed movements in the square and rectangular chambers than in the circular chamber. Second, heroin affected the contribution of these movements significantly more in the rectangular than in the square chamber.

Comparing the drug effects across chambers relative to saline effects across chambers illuminates the degree to which the environment is having an effect on the druginduced changes of movement patterns. First, nomifensine increased circumscribed movements across all chambers. This increase was proportionate to the increase observed across different environments for saline controls. Thus, the influence of the geometry of the environment is similar for both saline- and nomifensine-treated animals. Second, MBDB increased straight movements most robustly in the circular chamber. However, the effect of MBDB on movement patterns across chambers differs from that observed in saline controls. In particular, the strongest effect of MBDB on straight movements was found in the circular chamber, in which saline animals exhibited the lowest contribution of straight movements 


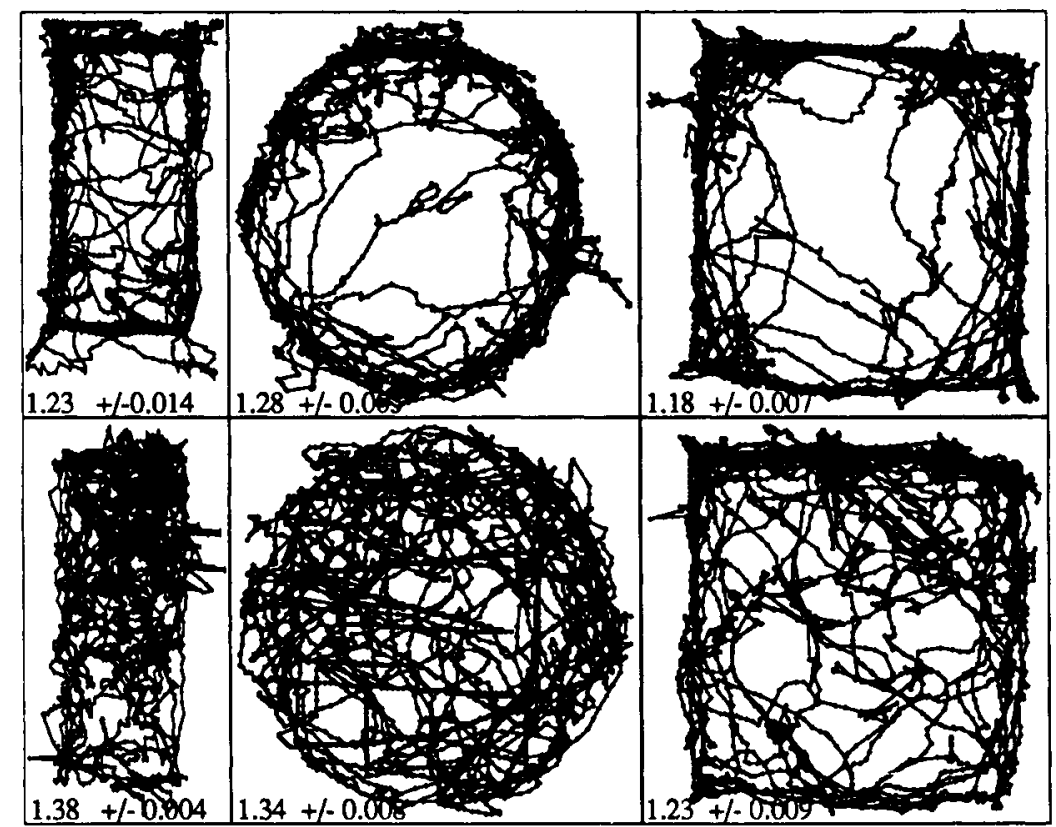

Figure 3. This figure shows the effect of saline (top three panels) or $5.0 \mathrm{mg}$ of nomifensine (bottom three panels) on movement patterns during 30 min of exposure to the rectangular, circular, or square chamber. The average spatial scaling exponent and the associated standard error are shown for each pattern.

relative to the other chambers. Thus, MBDB increases most strongly a movement pattern with the lowest baseline rate in saline animals. Third, heroin increased the contribution of circumscribed movements in the rectangular and square chambers but reduced their contribution in the circular chamber. The baseline rate of these movements in saline animals is significantly increased in the circular chamber over those in the rectangular and square chambers, suggesting that high baseline rates are reduced and low baseline rates are increased by heroin. These results apply to a lesser degree for straight movements. Here, the increase of straight movements induced by heroin was found to be most significant in the circular chamber, which corresponds to the lowest baseline rate of these movements in saline animals.

\section{SUMMARY AND DISCUSSION}

There are three main results of this investigation. First, movement patterns differ significantly across environments with different geometric properties. Second, druginduced changes of these movement patterns are similar across different environments. Third, the magnitude of drug-induced changes of these patterns can be either directly or inversely proportionate to the baseline rate observed in saline animals.

These results support several hypotheses. First, the organization of unconditioned motor behavior in rats underlying these movement patterns depends on the environmental properties. Second, the overall drug effect is similar in environments with different geometric properties. Third, the organization of unconditioned motor behavior is affected differentially by drugs, depending on the environmental properties. Fourth, the drug-induced changes in movement patterns observed using a videocamera-based system confirmed the pattern changes obtained in a photobeam system (Geyer \& Callaway, 1994; Paulus et al., 1993; Paulus \& Geyer, 1992).

First, investigating the effect of size, symmetry, and boundary characteristics of the environment on the detailed geometry of movements can provide a deeper understanding of how external stimuli influence behavioral organization of locomotor behavior. For example, straight and directed movements along a straight wall comprise a significant component of locomotor behavior found in animals exploring a novel environment. Moreover, a significant portion of locomotor behavior is characterized by meandering movements requiring a mild to moderate change in direction, as observed when animals move along the walls in the circular environment. Finally, exploratory and/or investigatory acts such as rearing or sniffing comprise locomotor behavior that is characterized geometrically by circumscribed movements.

Second, combining different environments and pharmacological manipulation of neural systems that are crucially involved in behavioral organization may provide insights into the adaptive nature of the pharmacologically induced behavioral changes. The particular drugs had been selected on the basis of previous results in the BPM (Geyer \& Callaway, 1994; Paulus et al., 1993; Paulus 
\& Geyer, 1992) and were known to differentially affect the geometrical characteristics of the movements. Specifically, pharmacological manipulation of neural systems may result in the repetitive and "purposeless" selection of locomotor behavior, which has been described as stereotypy. For example, continued forward movements or repetitive circumscribed movements have been described as stereotypic when induced by different drugs (Schiorring, 1971).

Movement patterns generated by saline controls in different environments presumably reflect the adaptive locomotor response to a novel situation and are thought to result from a selection and weighting process of available behavioral responses (Robbins, 1981). Comparing movement patterns generated by rats treated with a drug with those generated by saline controls in different environments provides information about the manipulation of the selection and weighting process underlying behavioral organization by the particular pharmacological agent. Alternatively, comparing movement patterns generated by rats treated with a drug in one environment with those generated by the same drug in a different environment may reveal the environment-related component of behavioral organization. Differential effects of drugs on movement patterns in different environments that parallel those observed in saline controls suggest a significant influence of the environment. In contrast, a lack of differential effects of drugs across different environments or differential effects that significantly deviate from those observed in saline controls suggest an environmentindependent behavioral organization or a significant change of the environmental influence on behavioral organization. Consequently, the effect of the drug can be characterized as environmentally dependent, environmentally independent, or environmentally idiosyncratic.

Specifically, nomifensine-treated animals exhibited relative contributions of straight movements in the different environments similar to those found for controls. In contrast, nomifensine affected the relative contributions of circumscribed movements in the different environments in a manner that was distinct from the differences observed with saline controls. Thus, the recruitment of behavioral elements characterized by circumscribed movements does not follow the adaptive pattern seen with saline controls. This dissociated pattern of behavior for circumscribed movements agrees with the observation that many indirect dopaminergic agonists produce local stereotypic movements such as head-bobbing or sniffing (Delfs \& Kelley, 1990).

MBDB treatment produced a pattern that is distinct not only in its geometry, as described in previous studies (Callaway et al., 1991; Geyer \& Callaway, 1994; Paulus et al., 1993), but also with respect to its adaptiveness when compared with nomifensine. Specifically, the contribution of straight movements differed substantially from that observed for saline controls. Not only did MBDB increase the contribution of these movements in the rectangular and circular environments relative to saline controls, but this increase was not significantly different for the different environments. Therefore, the behavioral organization associated with straight movements is dissociated from the differential stimuli imposed by the different geometrical properties of the chambers. This finding agrees with previous observations of the "march-like," stereotypic patterns observed with methylenedioxy-substituted amphetamines, for example, MDMA, MDA, or MBDB (Geyer \& Callaway, 1994; Paulus et al., 1993). Similarly, differential contributions of circumscribed movements in the different environments are unlike those observed for saline controls. In particular, environments containing corners, that is, the rectangular and square environments, resulted in significantly increased circumscribed movements. These results suggest that a sudden change in direction, as imposed by a corner, might lead to a relative increase in circumscribed movements. These movements possibly reflect a reorganization of behavioral elements. This interpretation would confirm anecdotal evidence from behavioral observation of animals treated with substituted amphetamines. When these animals reach a boundary that requires a fundamental change in movements, an interruption of the flow of movement, which may last several seconds, has been observed (unpublished observations, Geyer \& Callaway).

Lastly, heroin affected straight movements similarly across different environments and, when compared with controls, most significantly in the circular chamber, which is consistent with an environmentally nonadaptive pattern for these movements. In contrast, the significant change in circumscribed movements induced by heroin follows a pattern that differs from that observed in saline controls. Specifically, heroin increased circumscribed movements more significantly in the rectangular and square chambers than in the circular chamber. These findings are directly opposite to those for saline controls. These results suggest an environmentally idiosyncratic pattern for the effect of heroin on circumscribed movements. The increased contribution of circumscribed movements for saline animals within the rectangular and square chambers may suggest that the corners in these environments exert strong effects on behavioral organization. The results further support behaviorally the pharmacological evidence that heroin-induced locomotor activity is mediated by a neurobiological substrate distinct from that mediating dopaminergic hyperactivity (Amalric \& Koob, 1985; Swerdlow, Vaccarino, \& Koob, 1985).

There are several limitations to the studies presented here. First, the resolution of the Video Tracker instrument was reduced arbitrarily to match that of the previously described BPM. This reduction precludes the recording of very fine-scaled movements that may significantly impact measures of activity. However, the scaling approach uses the relationship between scales and movement activity and geometry to eliminate the scale-dependent char- 
acteristics of activity. Therefore, this restriction does not significantly affect the calculation of the scaling exponent. Second, the three environments used here differ across a number of specific environmental characteristics such as size or chamber area, symmetry, and boundary characteristics. However, ordering the chambers according to increasing size or area, symmetry, or number of corners and comparing the effects of saline, nomifensine, MBDB, and heroin on movement patterns across these dimensions yielded analogous findings and does not change the interpretation. These characteristics, however, will need to be examined in more detail in future investigations. Third, the results support the application of the extension of the scaling hypothesis to include a multifractal approach (Feder, 1988; Grassberger et al. 1988), since the average spatial scaling exponent did not reveal significant differences in five of the nine comparisons, whereas the extended approach was able to detect differences in eight out of nine comparisons. Fourth, the movement patterns quantified via the extended scaling approach are not simply related to observer-defined behaviors. Thus, caution should be taken when comparing these results with those from the observational studies of other investigators.

To summarize, the results presented here provide evidence for changes in behavioral organization of movements that are both associated and dissociated from the different geometries of the environment. Moreover, the differences are readily interpretable on the basis of behavioral observations of the animals. Lastly, the usage of different environments as well as statistics is readily understood as a consequence of the behavioral organization exhibited by the animals in these environments.

\section{REFERENCES}

ADAMS, L. M., \& GEYER, M. A. (1982). LSD-induced alterations of locomotor patterns and exploration in rats. Psychopharmacology, 77, $179-185$

AMALRIC, M., \& KоOB, G. F. (1985). Low doses of methylnaloxonium in the nucleus accumbens antagonize hyperactivity induced by heroin in the rat. Pharmacology, Biochemistry \& Behavior, 23, 411-415.

Callaway, C. W., Johnson, M. P., Gold, L. H., Nichols, D. E., \& GEYER, M. A. (1991). Amphetamine derivatives induce locomotor hyperactivity by acting as indirect serotonin agonists. Psychopharmacology, 104, 293-301.

Creese, I., \& Iversen, S. D. (1974). The role of forebrain dopamine systems in amphetamine-induced stereotyped behavior in the rat. Psychopharmacology, 39, 345-357.

DELFS, J. M., \& KeLLEY, A. E. (1990). The role of D1 and D2 dopamine receptors in oral stereotypy induced by dopaminergic stimulation of the ventrolateral striatum. Neuroscience, 39, 59-67.

DENENBERG, V. H. (1969). Open-field behaviour in the rat: What does it mean? In E. Tobach (Ed.), Experimental approaches to the study of emotional behavior (Vol. 1, Suppl. 1, pp. 163-174). New York: Ankho International.

Donat, P. (1991). Measuring behaviour: The tools and the strategies. Neuroscience \& Biobehavioral Reviews, 15, 447-454.

EILAM, D., \& GolANI, I. (1990). Home base behavior in amphetaminetreated tame wild rats (Rattus norvegicus). Behavioural Brain Research, 36, 161-170.

Feder, J. (1988). Fractals. New York: Plenum.

GEYER, M. A. (1982). Variational and probabilistic aspects of exploratory behavior in space: Four stimulant styles. Psychopharmacology Bulletin, 18, 48-51.

Geyer, M. A., \& Callaway, C. W. (1994). Behavioral pharmacology of ring-substituted amphetamine analogs. In A. K. Cho \& D. S. Segal (Eds.), Amphetamine and its analogs: Neuropsychopharmacology, toxicology and abuse (pp. 177-208). New York: Academic Press.

Geyer, M. A., Russo, P. V., \& Masten, V. L. (1986). Multivariate assessment of locomotor behavior: Pharmacological and behavioral analyses. Pharmacology, Biochemistry \& Behavior, 25, 277-288.

Grassberger, P., Badi, R., \& Politi, A. (1988). Scaling laws for invariant measures on hyperbolic and nonhyperbolic attractors. Journal of Statistical Physics, 51, 135-178.

Halsey, T. C., Jensen, M. H., Kadanoff, L. P., Procaccia, I., \& Shraiman, B. I. (1986). Fractal measures and their singularities: The characterization of strange sets. Physical Review A, 33, 1141-1151.

HigusHI, T. (1988). Approach to an irregular time series on the basis of fractal theory. Physica $D, 31,277-283$.

LAT, J. (1965). The spontaneous exploratory reactions as a tool for psychopharmacological studies. A contribution towards a theory of contradictory results in psychopharmacology. In M. Y. Mikhelson, U. G. Longo, \& Z. Votava (Eds.), Pharmacology of conditioning, learning and retention (pp. 47-66). Oxford: Pergamon Press.

LJUNGBerG, T., \& ENQUist, M. (1987). Disruptive effects of low doses of d-amphetamine on the ability of rats to organize behaviour into functional sequences. Psychopharmacology, 93, 146-151.

MaNdelbrot, B. (1977). Fractals: Form, chance and dimension. San Francisco: Freeman.

Mandell, A. J., \& Selz, K. A. (1997). Entropy conservation as $h_{T u} \approx$ $\lambda_{\mu}+d_{\mu}$ in neurobiological dynamical systems. Chaos, 7, 67-81.

MCREy NOLDS, P. (1962). Exploratory behavior: A theoretical interpretation. Psychological Reports, 11, 311-318.

MonTGomery, K. C. (1955). The relation between fear induced by novel stimulation and exploratory behavior. Journal of Comparative Physiology \& Psychology, 48, 254-260.

Mueller, K., Hollingsworth, E. M., \& Cross, D. R. (1989). Another look at amphetamine-induced stereotyped locomotor activity in rats using a new statistic to measure locomotor stereotypy. Psychopharmacology, 97, 74-79.

NicKolson, V. J. (1981). Detailed analysis of the effects of apomorphine and d-amphetamine on spontaneous locomotor behaviour of rats as measured in a TV-based automated open-field system. European Journal of Pharmacology, 72, 45-56.

NORTON, S. (1977). The study of sequences of motor behavior. In L. L. Iversen, S. D. Iversen, \& S. H. Snyder (Eds.), Handbook of psychopharmacology (Vol. 7, pp. 83-105). New York: Plenum.

Paulus, M. P., Callaway, C. W., \& Geyer, M. A. (1993). Quantitative assessment of the microstructure of rat behavior: II. Distinctive effects of dopamine releasers and reuptake inhibitors. Psychopharmacology, 113, 187-198.

Paulus, M. P., \& Geyer, M. A. (1991). A temporal and spatial scaling hypothesis for the behavioral effects of psychostimulants. Psychopharmacology, 104, 6-16.

Paulus, M. P., \& Geyer, M. A. (1992). The effects of MDMA and other methylenedioxy substituted phenylalkylamines on the structure of rat locomotor activity. Neuropsychopharmacology, 7, 15-31.

Paulus, M. P., \& Geyer, M. A. (1993a). Quantitative assessment of the microstructure of rat behavior: $I . f(d)$, the extension of the scaling hypothesis. Psychopharmacology, 113, 177-186.

Paulus, M. P., \& Geyer, M. A. (1993b). Three independent factors characterize spontaneous rat motor activity. Behavioral Brain Research, 53, 11-20.

RoBbins, T. W. (1977). A critique of the methods available for the measurement of spontaneous motor activity. In L. L. Iversen, S. D. Iversen, \& S. H. Snyder (Eds.), Handbook of psychopharmacology (Vol. 7, pp. 37-82). New York: Plenum.

RobBins, T. W. (1981). Behavioural determinants of drug action: Ratedependency revisited. In S. J. Cooper (Ed.), Theory in psychopharmacology (Vol. 1, pp. 1-63). New York: Academic Press.

RUELLE, D. (1984). Thermodynamic formalism: The mathematical structures of classical equilibrium statistical mechanics. New York: Cambridge University Press. 
SANBERG, P. R., Zoloty, S. A., Willis, R., Ticarich, C. D., Rhoads, K., NaGy, R. P., Mitchell, S. G., Laforest, A. R., Jenks, J. A., \& HARKABUS, L. J. (1987). Digiscan activity: Automated measurement of thigmotactic and stereotypic behavior in rats. Pharmacology, Biochemistry \& Behavior, 27, 569-572.

SCHIORRING, E. (1971). Amphetamine induced selective stimulation of certain behaviour items with concurrent inhibition of others in an open-field test with rats. Behaviour, 29, 1-17.

STANLEY, H. E. (1987). Introduction to phase transitions and critical phenomena. New York: Oxford University Press.

Swerdlow, N. R., Vaccarino, F. J., \& KoOB, G. F. (1985). Effects of naloxone on heroin-, amphetamine- and caffeine-stimulated locomotor activity in the rat. Pharmacology, Biochemistry \& Behavior, 23, 499-501.

Szechtman, H., Ornstein, K., Teitelbaum, P., \& Golani, I. (1985).
The morphogenesis of stereotyped behavior induced by the dopamine receptor agonist apomorphine in the laboratory rat. Neuroscience, 14, 783-798.

Teitelbaum, P., Szechtman, H., Sirkin, D. W., \& Golani, I. (1982). Dimensions of movement, movement subsystems and local reflexes in the dopaminergic systems underlying exploratory locomotion. In M. Y. Spiegelstein \& A. Levy (Eds.), Behavioral models and the analysis of drug action (pp. 357-385). Amsterdam: Elsevier.

Weiss, F., Paulus, M. P., Lorang, M. T., \& Koob, G. F. (1992). Increases in extracellular dopamine in the nucleus accumbens by cocaine are inversely related to basal levels: Effects of acute and repeated administration. Journal of Neuroscience, 12, 4372-4380.

(Manuscript received February 11, 1997; revision accepted for publication September 17, 1997.) 